\title{
Autonomous on-Chip Debugging for Sensors Based on AVR Microcontrollers
}

\author{
Antoine Bossard \\ Graduate School of Science, Kanagawa University, Kanagawa, Japan \\ Email: abossard@kanagawa-u.ac.jp
}

How to cite this paper: Bossard, A. (2021) Autonomous on-Chip Debugging for Sensors Based on AVR Microcontrollers. Jour nal of Sensor Technology, 11, 19-38. https://doi.org/10.4236/jst.2021.112002

Received: May 3, 2021

Accepted: June 18, 2021

Published: June 21, 2021

Copyright (c) 2021 by author(s) and Scientific Research Publishing Inc. This work is licensed under the Creative Commons Attribution International License (CC BY 4.0).

http://creativecommons.org/licenses/by/4.0/

\begin{abstract}
Sensors are often based on minimalistic microcontrollers for their reduced power consumption and size. Because of the specific hardware of sensors, their software development, including debugging, is also particular. Simulators and external computers are conventional approaches to sensor debugging, but they both face limitations such as the supported hardware and debugging conditions. In this paper, we propose a fully autonomous on-chip debugging solution for sensors (and other devices) based on AVR microcontrollers, with a particular focus on human-machine interaction. The proposal is then validated in practice through various experiments, notably involving real-world sensors. Formal measurement of the induced overhead is also conducted, which eventually demonstrates the applicability of the proposal.
\end{abstract}

\section{Keywords}

RISC, Computer, Architecture, ISA, Software, Assembly, Electronics, Green Computing, HMI

\section{Introduction}

Comparable to some embedded systems, sensors need to be efficient with respect to their power consumption, especially when used in a mobile environment or the Internet of things (IoT), and this is directly related to the space required by the sensor hardware [1]. As a result, such devices are often based on particular chips that can help satisfying these requirements. The microcontrollers that implement the AVR or ARM processor architectures are examples of such chips [2] [3]. The Arduino Uno and BBC Micro Bit devices are concrete examples of devices based on such microcontrollers, whereas a device such as the Raspberry Pi features a more powerful processor-it is the system on a chip (SoC) approach [4] [5] [6]. 
Besides, as such devices, sensors, often rely on specific hardware components notably to implement their sensing features [7], their software development is very specialised in the sense that the microcontroller program needs to interact with the hardware components, with each having its own specificities like I/O control and analog-to-digital conversion. Furthermore, because we rely here on low-level programming, there is a notable lack of interfacing standardization across those peripherals, something which is commonly realised by the architecture layers of the operating system in the case of more advanced computer systems. The large number of available libraries for sensors used with an Arduino board is a good indicator of this situation. And obviously, a debugging solution that is able to adapt itself to such a specialised software development is required as well [8].

We propose in this paper a fully autonomous on-chip debugging (OCD) solution for sensors and other devices that are based on AVR microcontrollers. This approach particularly enhances human-machine interaction for the debugging of sensors. In simple terms, its principle is as follows: a liquid crystal display (LCD) panel is connected to the board with a minimally invasive method and each microcontroller (processor) instruction of the original program (i.e. of the program being debugged) that is about to be executed is displayed in real-time on the LCD, just before its execution. This is a rather natural approach but its realisation is highly challenging in several aspects as detailed hereinafter. For instance, a debugging solution like the one proposed in this paper cannot be realised by simply reading (parsing) assembly instructions from the machine code for the microcontroller and then using a conventional program, say, written in the $\mathrm{C}$ language to print them on the display. The objective of this research is to show on the display real-time information about the processor instruction that is about to be executed, and this cannot be achieved with a conventional approach based, say, on a program written in $\mathrm{C}$, and the classic toolchain (e.g. the development framework that corresponds to the selected hardware). The merit of the proposal compared to the conventional approaches is further discussed in Section 2.

This work started from the following fact: as computers are increasingly complex devices, one of the most recurrent HMI issues is when the system fails to respond. This is usually a frustrating situation as the state of the machine remains unknown to the user. Aiming at investigating further this issue and possible solutions, we have selected a simple device, a microcontroller, and some of its applications, sensors, and we describe hereinafter our approach which fully resolves this HMI issue: at any time, the state of the device is known. The motivation and contribution of our proposal with respect to the sensor HMI issue is further explained in the next section.

The rest of this paper is organised as follows. Previous and related works are discussed in Section 2, notably to emphasise the contribution of the proposal. Then, preliminaries for the proposal are detailed in Section 3. Next, the proposed debugging system is detailed in Section 4. It is then validated in practice in Sec- 
tion 5 and formally evaluated in Section 6 . The obtained results are subsequently discussed in Section 7. Finally, this paper is concluded in Section 8.

\section{State-of-the-Art and Contribution}

Regarding previous and related works, simulators are one conventional approach to debugging for such microcontrollers. They are numerous and available on various operating systems; Paulware Arduino Debugger [9] and UnoArduSim [10] are two examples of such works. Unlike those, the research described and evaluated in this paper realises "live", in situ debugging, that is directly on the chip, with thus the great advantage of being able to handle any program that uses any kind of sensor, and hardware in general, which a conventional simulator would not support and would hence not be able to debug such a program properly. Concretely, simulators usually give a list of the supported libraries: for instance, UnoArduSim only supports the TFT, SD, Stepper, Servo, SoftwareSerial, Wire, SPI, and EEPROM libraries out of the official, standard thirteen libraries [11].

More closely related to our work, some approaches such as those of [12] and [13] collect debugging information directly from the microcontroller but then need additional processing by an external computer. Compared to our proposal, those approaches are not autonomous, and they suffer from a very high cost: those methods are in fact very demanding with respect to the required additional hardware and the necessary software environment needed to process the collected debugging information. Besides, these works are yet another proof of the relevance of on-chip debugging. The development environment provided by the microcontroller manufacturer, Microchip Studio for AVR and SAM devices (formerly Atmel Studio) and notably its debugger component [14] (see also [15]), and the portable debugger GNU Debugger (GDB) [16] are other examples of debugging solutions that follow this conventional approach, and with its limitations.

In addition, from the system security point of view, it is worth noting that our proposal relies neither on a JTAG interface as [17] and the previously cited works [12] and [13] nor on permanent debugging (and diagnosis) code, two exploitation vectors pointed out for instance in [18]. This security issue induced by the JTAG interface is also mentioned in the work described in [19] (and in [20]), work which however still has a very high cost for the reasons given in the previous paragraph. A similar debugging approach, and with thus the same limitations, is provided by some ATmega microcontrollers with the debugWIRE system [21] [22]. Furthermore, unlike such an approach, and although some minor adjustments might be needed, our approach is applicable to any microcontroller based on the AVR instruction set architecture. In fact, the proposed debugging system is universal in that it is applicable to any AVR program, no matter how it has been produced (compiled from $\mathrm{C}$, assembled, etc.).

We next further explain the motivation and contribution of our proposal with respect to the HMI issue. The time (duration) and place of the debugging process 
is another reason for the high cost mentioned for the conventional approach based on an external, supplemental device specially used for debugging. The essence of the proposed system is about being "in situ", which means of course onchip debugging, but also that the sensor should be debugged and monitored in real conditions. Here, time and place are two essential such conditions which relate to on-site deployment and on-site testing of the sensor device.

Time, for the duration of the debugging and monitoring process: the sensor system should be in use for a long time enough, and needs to be easily and regularly checked (e.g. [23]). This is not possible with an external computer used for debugging: even if that supplemental computer can be used for debugging automatically for a long time, its monitoring can only be done regularly on a restricted time interval as the programmer can only stay a limited time in front of it. With the proposed system, since it implements a fully autonomous debugging solution, the sensor device can stay at all times near the programmer, like at home, for monitoring on wide time intervals.

And the place (location) of the sensor device is even more critical: it needs to be used in its real environment otherwise it makes little sense to do the sensing (e.g. air quality monitoring [24]). This is again not possible with an external computer used for debugging; the reader can simply imagine designing a sensor device for transportation systems (thus aimed at a mobile environment), domestic usage, and so on: the external computer for debugging cannot reasonably be carried and placed nearby the sensor device in real conditions.

\section{Preliminaries}

A schematic of a sample circuit is given in Figure 1; the one used in this research is based on an Arduino Uno Rev3, which is itself based on a 28 PDIP ATmega328P microcontroller, coupled with an HD44780U-compatible LCD panel operating at $5 \mathrm{~V}$. The pins that correspond to the three ports $\mathrm{B}, \mathrm{C}, \mathrm{D}$ of the microcontroller are emphasised. This is a rather conventional usage of an HD44780Ucompatible LCD panel in the 4-bit operation mode (i.e. using four data bus pins:

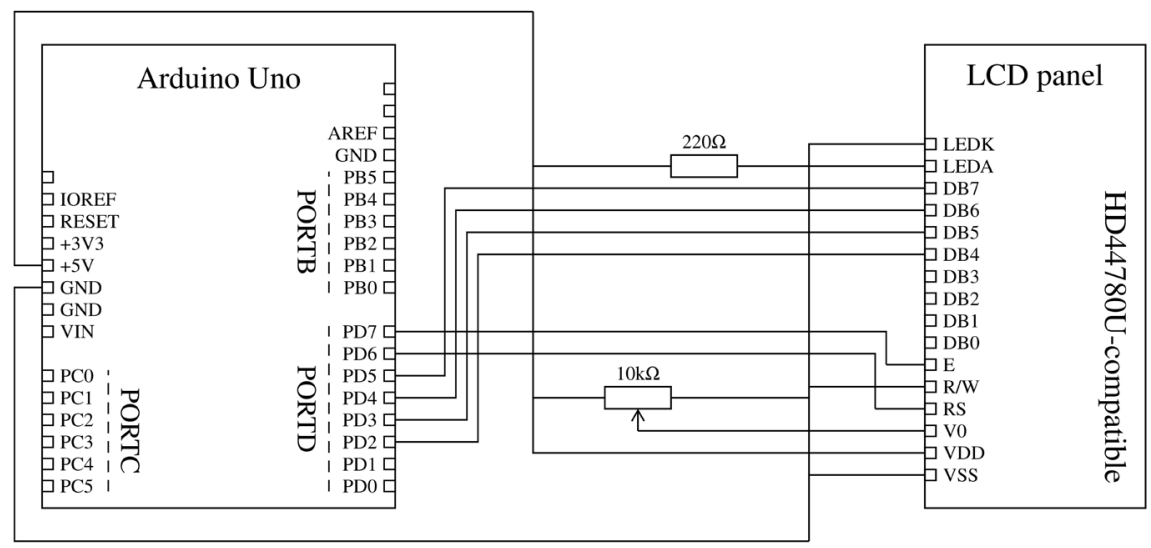

Figure 1. Schematic of a sample circuit: the one used in this research is based on an Arduino Uno Rev3, which is itself based on a 28 PDIP ATmega328P microcontroller. 
DB4 to DB7), yet it can be noted that all connections are smartly gathered at one single port (D) in order to minimise the number of pins reserved for debugging. Six pins of the microcontroller are thus allocated to (i.e. reserved for) the debugger, which is optimally low considering the hardware involved. We on purpose leave the two pins 0 and 1 of port $\mathrm{D}$ available as they are used for the board's serial communication (RX and TX, respectively), a communication feature which, although not used by the proposed debugging system, may well be used by the program to be debugged. Since no data is read from the LCD panel, only written to it, the R/W pin of the LCD panel is directly wired to the earth (i.e. $\mathrm{R} / \mathrm{W}$ to 0 so as to set the LCD panel in write mode). An overview of the actual prototype is given in Figure 8.

\section{On-Chip Debugging}

The principle of the proposed on-chip debugging system for sensors based on AVR microcontrollers is illustrated with a flow chart in Figure 2: the proposed system consists of two main components, the disassembler and the injector, which are detailed hereinafter. The dashed lines on the right side of the diagram indicate abbreviation of the input and output objects for the sake of readability. A HEX file contains ASCII byte code as per the Intel HEX standard [25]. The HEX file of the original program thus represents the machine code of the program that will be subject to debugging. The final HEX file is eventually uploaded into the microcontroller to be run (to this end, we rely on the default bootloader and avrdude (version 6.3)).

For reference, we also give in Figure 3 a simplified UML class diagram of the proposed system as it provides relevant information to facilitate the understanding of the system description given in the rest of this section. Most notably, this class diagram shows the non-trivial relations which involve the main Disassembly class. An object of this class can in fact either be instantiated from one Hexbytes object (representing one HEX file) - this is the disassembler use case, or from three Disassembly objects-this is the injector use case.

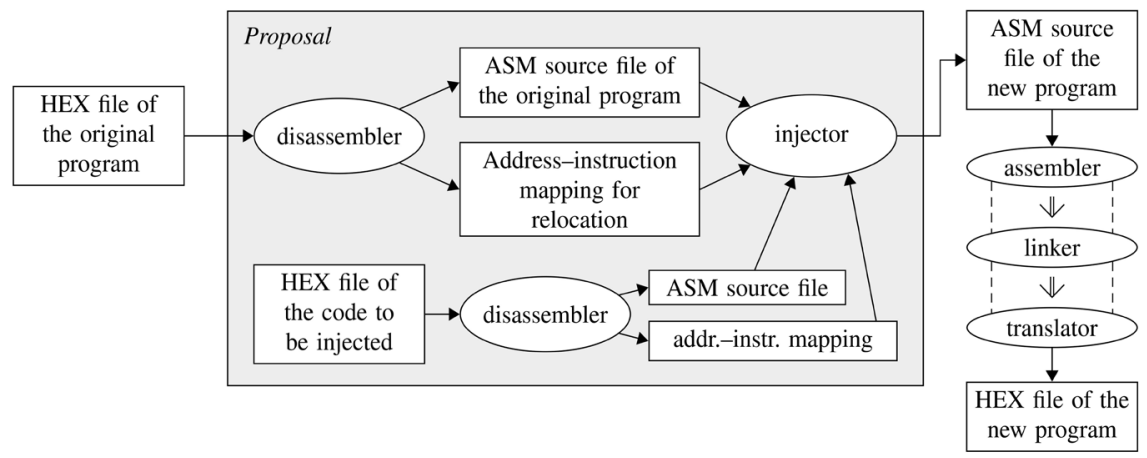

Figure 2. Flow chart illustrating the principle of the proposed debugging system. (The dashed lines on the right side of the diagram indicate abbreviation of the input and output objects.) 


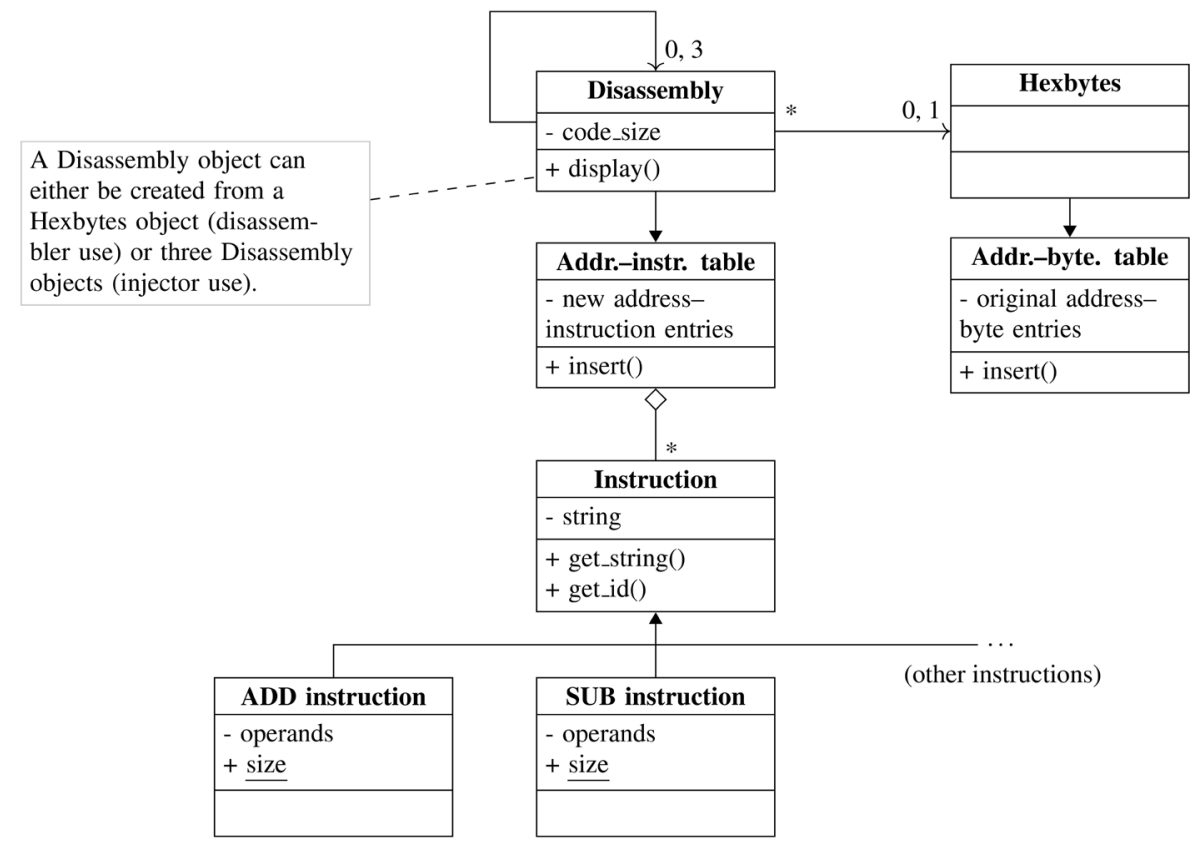

Figure 3. Simplified UML class diagram of the proposed debugging system. It notably shows the non-trivial relations that involve the main Disassembly class.

The other software tools used in this research are based on the GNU Binutils (version 2.26): the assembler is avr-as, which is part of the GNU as assembler collection; the linker is avr-ld, from GNU ld; the translator is avr-objcopy, from GNU objcopy. As briefly explained previously, the HEX file of the original program can have been produced by various means: compiled from $\mathrm{C}$ with avr-gcc, assembled from ASM with avr-as, and so on.

\subsection{The Disassembler Component}

It is recalled that reverse engineering of machine code for such microcontrollers is limited to assembly code. Assembly code in fact maintains a 1-to-1 correspondence to the machine code (thus comparable to a bijective function in mathematics), or almost (e.g. there are instruction "synonyms"). However, it is not possible to reverse machine code back to, for instance, the original code written with the $\mathrm{C}$ language since compilation of $\mathrm{C}$ code to machine code loses information: there is no 1-to-1 mapping between $\mathrm{C}$ code and machine code.

Although, as presented in Section 2, there exist several disassemblers for AVR programs [14] [15] [16] [26] [27] [28], as this research project requires very low-level manipulation of machine code instructions and their addresses (especially for the injector component of the proposed system that is detailed in the next section), we have based the disassembler component of the proposal on our own AVR disassembler.

First, the HEX file of the original program (i.e. the program that is to be debugged) is parsed by reading Intel HEX records [25]: from this process is obtained an address-byte table (a map; if needed, the bytes are reordered according 
to their addresses, although usually the byte order matches the addresses declared in the HEX file). Here, the addresses are byte-based.

Second, the entries of this address-byte table are iterated two-by-two in order: two consecutive bytes are merged since an AVR instructions on 8-bit AVR devices consists of one or two words [29]. Bits of the first word (i.e. the first two bytes) are thus used to identify the instruction's opcode and operands. The next word is read when the identified instruction is double-word, typically instructions that involve a full, 16-bit memory address such as CALL. During this process, the word-based address of the instruction is calculated and its operands, if any, are retrieved. As a result, an address-instruction table (map) is obtained; this table is a perfect match of the instructions, and thus another representation, of the original program.

The source of a sample original program-then assembled-and the corresponding address-instruction table that is output by the disassembler component are shown in Figure 4(a) and Figure 4(b), respectively. In the latter figure, there are two address columns: the leftmost one is for the new, possibly recalculated instruction address, and the second one shows the original address of the instruction. Since we illustrate in this section the disassembler component on its own, the two addresses are equal for each table entry (i.e. instruction). This sample source code shows typical microcontroller initialisation instructions and a loop example: it can be noticed that the relative jump offset of the branching instruction BRNE is automatically calculated by the assembler, which stores it in words; the disassembler then restores it in bytes and prints it in accordance with the assembler (avr-as) syntax.

Finally, it should be noted that, as illustrated in Figure 2, not only is the disassembler component applied to the machine code of the original program, but it is also applied to the machine code of the debugger that will be further processed by the injector component as detailed next.

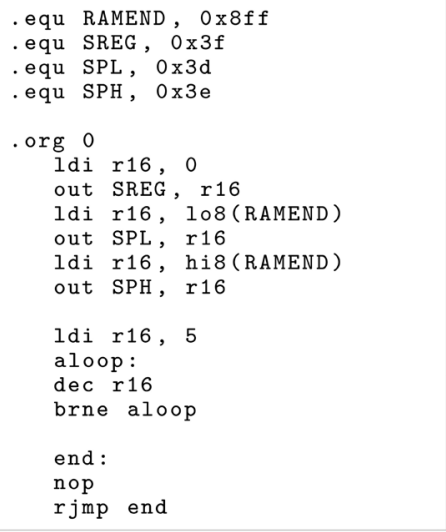

(a)

\begin{tabular}{|c|c|c|}
\hline [new addr] & [orig addr] & instruction \\
\hline [0x0000] & [0x0000 orig] & ldi r16, 0 \\
\hline [0x0001] & [0x0001 orig] & out $0 \times 3 f, r 16$ \\
\hline [0x0002] & [0x0002 orig] & ldi $r 16,255$ \\
\hline [0x0003] & [0x0003 orig] & out $0 \times 3 d, r 16$ \\
\hline [0x0004] & [0x0004 orig] & ldi $r 16,8$ \\
\hline [0x0005] & [0x0005 orig] & out $0 \times 3 e, r 16$ \\
\hline [0x0006] & [0x0006 orig] & ldi $r 16,5$ \\
\hline [0x0007] & [0x0007 orig] & dec $r 16$ \\
\hline [0x0008] & [0x0008 orig] & brne.-4 \\
\hline [0x0009] & [0x0009 orig] & nop ; $\Leftrightarrow$. byte ox \\
\hline [0x000a] & [0x000a orig] & rjmp. -4 \\
\hline
\end{tabular}

(b)

Figure 4. (a) The source code of a sample original program-then assembled-and (b) the corresponding address-instruction table that is output by the disassembler component of the proposed debugging system. 


\subsection{The Injector Component}

The second major part of the proposal is the injector component, whose purpose is to carefully intermingle the original program instructions with new instructions for debugging. To this end, the recalculation of the addresses used by the original program instructions, for instance destination addresses for absolute calls, jumps and offsets for relative ones, is a tedious yet critical task-a program for, say, sensor control, can hardly be considered without such calls and jumps as they make the logic of the program.

As shown in Figure 2, the injector component takes as input the instructions of the original program, together with their (original) addresses, as well as the instructions of the debugger code. It produces a new assembly source file that is to be assembled as usual (avr-as), then linked and converted to machine code in the Intel HEX format. Then, it is uploaded to the microcontroller board for execution in what we can call debugging mode. More precisely, the injector's input is split into the initialisation code of the debugger, which makes the first part of the output assembly file, thus comparable to a prefix to the program being debugged, and the code of the debugger (called the step code) that is to be injected at each instruction of the original program.

The injection process is done in two passes: the first one is to inject instructions and calculate their new addresses. The second one is to recalculate the addresses used by the original program instructions (e.g. calls, jumps). This process in two passes described below is rather complicated, thus its outline is also illustrated with the flow charts of Figure 5 and Figure 6, respectively for the first and second pass.

The first pass is done as follows. Beginning with an empty output file, the injector starts by orderly injecting each of all the instructions of the debugger initialisation code. The initial addresses match those newly calculated by the injector. Then, for each of all the instructions of the program being debugged, 1) the instructions of the debugger's step code are orderly injected, each time incrementing the address counter accordingly. Hence, the initial addresses of the step code do not match those newly calculated by the injector. And 2) the current instruction of the program being debugged is injected and its new address is calculated.

As a special case, if the instruction of the original program is OUT to the port $\mathrm{D}$, then the injector discards the original program bits for the debugging pins (2 to 7) so as not to jeopardise the debugging process, that is, retains only the bits for the two pins of this port unused by the debugger ( 0 and 1$)$. In practice, this is a rather complicated process which involves injecting several instructions for saving and restoring the state of the pins used by the debugger. This is also critical that the debugging system retains the value of the pins 0 and 1 of the port $\mathrm{D}$ since these two pins are used for serial communications, possibly by the program being debugged but not only: changing them would, for instance, hamper the upload of machine code to the board with avrdude. And otherwise, if the instruction of the original program needs to be skipped (e.g. since it would modify a memory location that is reserved for the debugging system), it is replaced by 


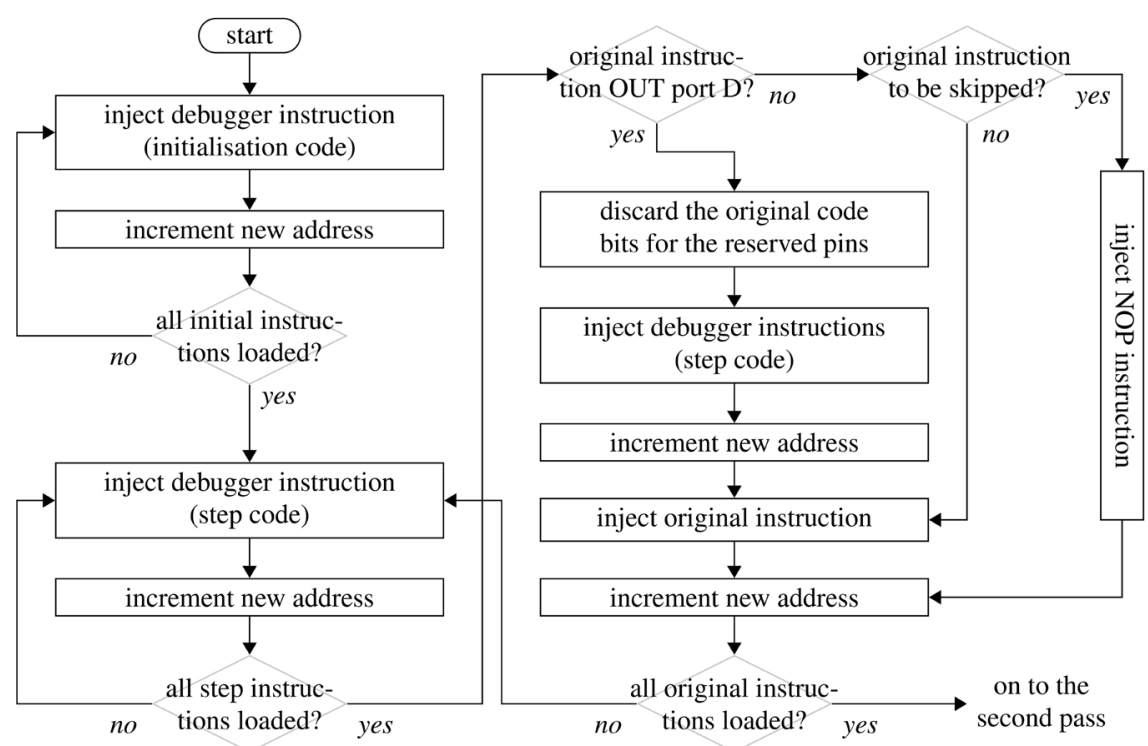

Figure 5. Flow chart outlining the first pass of the injector component of the proposed debugging system. It is connected to that of the second pass (see Figure 6).

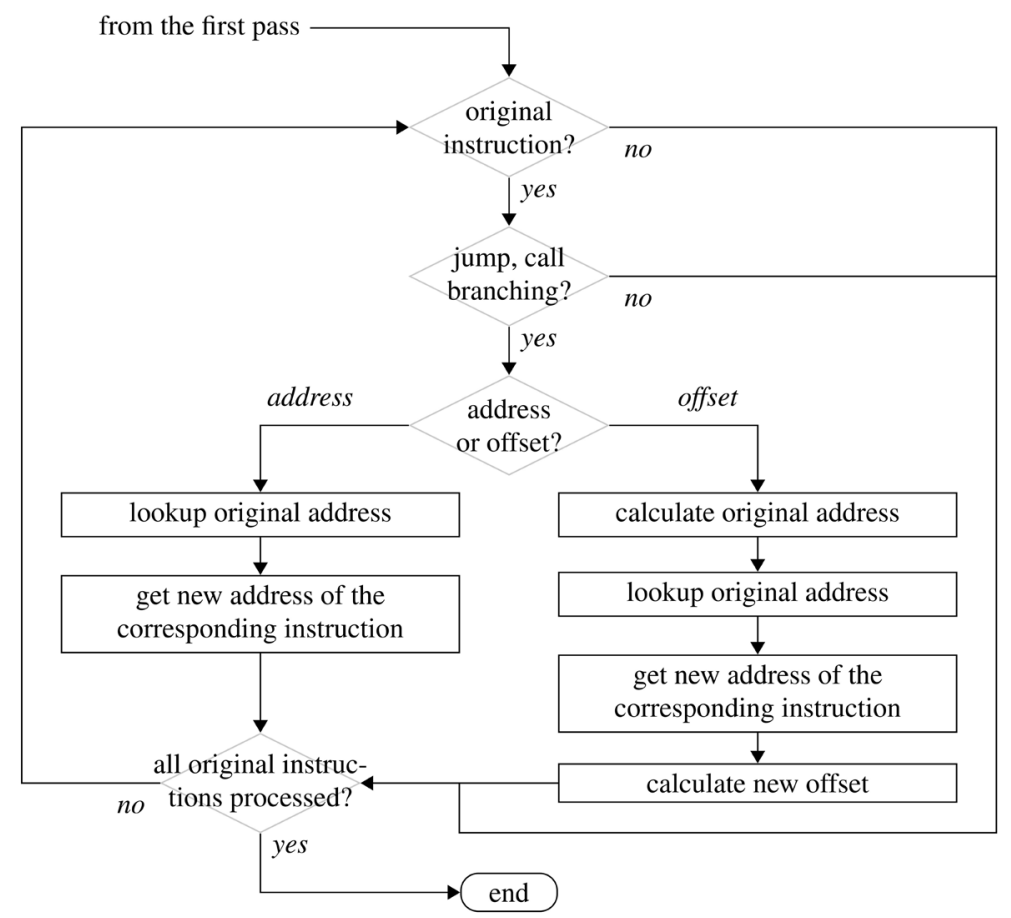

Figure 6. Flow chart of the second pass of the injector component of the proposed debugging system. It is connected to that of the first pass (see Figure 5).

the NOP instruction (i.e. "no operation"). Instructions such as SBRC are handled similarly as special cases; their details are here omitted for the sake of conciseness.

The second pass, which is executed once the first pass is complete, that is when all the instructions-original and for debugging-have been injected, is done as follows. For each of all the injected original instructions, and in ascending or- 
der of their newly calculated addresses, say $A_{i}^{\prime}$, check if the opcode is that of either a call, jump or branching instruction. In the case the call, jump destination is an absolute address (thus an original, possibly outdated instruction address), ${ }^{1}$ lookup this original destination address, say $A_{d}$, inside the address-instruction table (it holds for each instruction both the new and original addresses) to identify the corresponding instruction, say $I_{d}$. Then, get the newly calculated address of the instruction $I_{d}$, say $A_{d}^{\prime}$, which is thus the newly calculated destination address, i.e. that of $I_{d}$ the instruction pointed at by the call, jump, branching instruction.

In the case the call, jump, branching destination is a relative offset (thus an original, possibly outdated offset), ${ }^{2}$ say $k$, add the original offset $k$ to the original address, say $A_{i}$, of the call, jump, branching instruction to obtain the original destination address, say $A_{d}$ (precisely, we have $A_{d}=A_{i}+k+1$ to match [29]). Lookup $A_{d}$ inside the address-instruction table to identify the corresponding instruction, say $I_{d}$. Then, get the newly calculated address of the instruction $I_{d}$, say $A_{d}^{\prime}$, which is thus the newly calculated destination address, and deduce the new offset $k^{\prime}$ by subtracting from $A_{d}^{\prime}$ the (newly calculated) address $A_{i}^{\prime}$ of the current instruction (precisely, we have $k^{\prime}=A_{d}^{\prime}-A_{i}^{\prime}-1$ to match [29]).

The reader may have noticed that the injector outputs several (three, precisely) types of instructions:

Type 1: the instructions loaded from the original, debugged program's machine code;

Type 2: the instructions loaded from the debugger machine code (e.g. instructions of the step code);

Type 3: the new instructions dynamically created upon needs, for instance when the original program accesses the port $\mathrm{D}$. So, these new instructions depend on the original program code but also on the debugger step code.

Part of a sample injector output, notably showing the above instruction types, is illustrated in Figure 7; this is the part near the dec r16 instruction of the sample code of Figure 4(a). In this figure, the above type 1 is denoted by orig, the above type 2 by load and the above type 3 by crea. Besides, the difference between the original and new addresses can now be seen; the original address is 0 for the dynamically created instructions (i.e. type 3 above).

Finally, it should be noted that the injector reserves the last byte of the data memory space in SRAM. Let us also mention that although the CALL (long call) and JMP (long jump) instructions are supported by the proposed debugging system, because these instructions are not supported on all AVR devices [29], they are not employed by the proposal.

\section{Validation and Application to Real-World Sensors}

In this section, we show the validity of the proposed debugging system through ${ }^{1}$ The CALL or JMP instructions.

${ }^{2}$ The RCALL, RJMP, BRNE, etc. instructions. 
various experiments that show concrete operation of the on-chip debugging feature together with actual sample electronic circuitry for sensors.

As a validation experiment, we show an excerpt of the debugging result when applied to the sample original program of Figure 4(a). We have set a delay of the order of one second between the debugging information displayed for each instruction of the original program. A capture of the state of the debugging system is illustrated in Figure 8.

Next, we consider real-world sensor applications: first, we realise a simple light sensor (photodetector) based on an HW5P-1 phototransistor. The corresponding sensor control program simply turns on the LED embedded onto the board (pin 5 of port B) when the value read by the photodetector is higher than the threshold (see Figure 9(a)). This is achieved by connecting the phototransistor to one analog pin of the microcontroller, say $\mathrm{ADC} 0$, and accessing the value output by the analog-to-digital converter (ADC) that is provided by the microcontroller. When the photodetector is covered with some opaque material, the read value becomes smaller than the threshold and the embedded LED is not lit (see Figure 9(b)).

\begin{tabular}{|c|c|c|c|}
\hline new address $\square$ & dress $\downarrow$ & $\downarrow$ & nstruction type \\
\hline [0x0299] & {$[0 \times 000 f$} & load] & pop r25 \\
\hline [0x029a] & {$[0 \times 0010$} & load] & pop r16 \\
\hline$[0 \times 029 b]$ & {$[0 \times 0011$} & load] & out $\theta \times 3 f$, ro \\
\hline$[0 \times 029 c]$ & {$[0 \times 0012$} & load] & pop ro \\
\hline [0x029d] & {$[0 \times 0007$} & orig] & dec r16 \\
\hline [0x029e] & {$[0 \times 0000$} & load] & push ro \\
\hline [0x029f] & {$[0 \times 0001$} & load] & in ro, $0 \times 3 f$ \\
\hline$[0 \times 02 a \theta]$ & {$[0 \times 0000$} & crea] & rcall . -590 \\
\hline$[0 x 02 a 1]$ & {$[0 \times 0003$} & load] & push r31 \\
\hline$[0 \times 02 a 2]$ & {$[0 \times 0004$} & load] & push r30 \\
\hline$[0 \times 02 a 3]$ & {$[0 \times 0000$} & crea] & $1 d i r 31,0$ \\
\hline$[0 \times 02 a 4]$ & {$[0 \times 0000$} & crea] & Idi $r 30,232$ \\
\hline
\end{tabular}

Figure 7. Part of a sample injector output, notably showing the three types of injected instructions. Besides, the original and new addresses now differ.
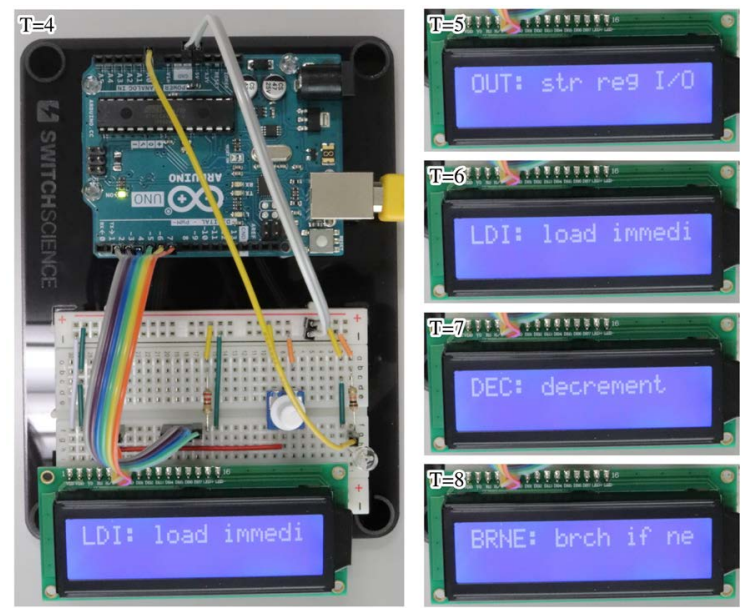

Figure 8. Successive photographs of the debugging system when applied to the sample original program of Figure 4(a) (excerpt). A time indicator T (in seconds) is given in the top left-hand corner of each photograph. 


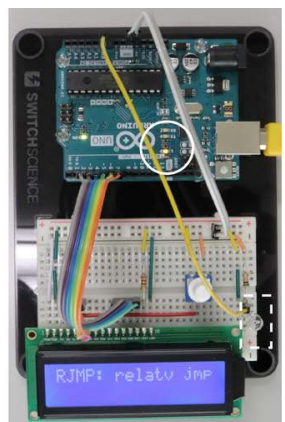

(a)

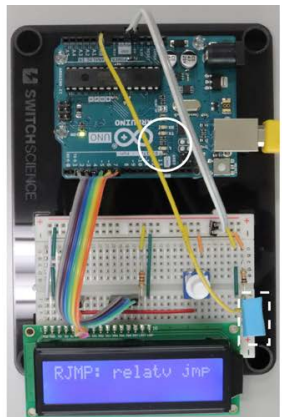

(b)

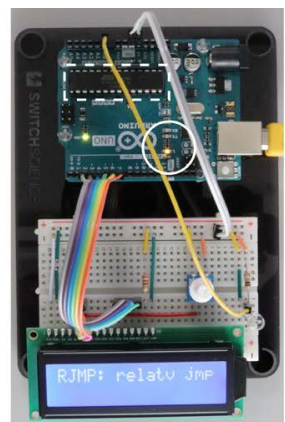

(c)

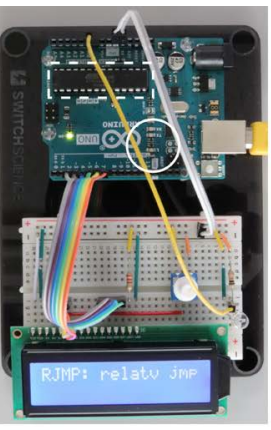

(d)

Figure 9. Application to real-world sensors: $(a, b)$ a photodetector and (c, d) a temperature sensor. When the value read by the phototransistor is higher than the threshold, the embedded LED (circled) is lit (a), and otherwise not (b) - it can be noticed that the phototransistor (dashed) is covered with an opaque material in the latter situation. When the value read by the temperature sensor (dashed; included inside the microcontroller) is higher than the threshold, the embedded LED (circled) is lit (c), and otherwise not (d).

Second, we realise a simple temperature sensor device which relies on the internal temperature sensor of the ATmega328P microcontroller (input channel ADC8 of the microcontroller ADC). The corresponding sensor control program simply turns on the embedded LED when the value read by the temperature sensor is higher than the threshold (see Figure $9(\mathrm{c})$ ). And when the value read by the temperature sensor is smaller than the threshold, then the embedded LED is not lit (see Figure 9(d)).

\section{Results: Overhead Measurement}

We show in this section the applicability of the proposal by formally measuring the induced memory size and performance overheads.

First, we measure the memory size overhead that is induced by the proposed debugging system. The values given below are in bytes. Since it depends on the microcontroller, we define $s$ the number of instruction types that have been implemented in the debugging system (typically, a few dozens of instruction types, and at most 119 [29]). The initialisation code requires a total of

$$
372+18 s
$$

bytes and the step code 42 bytes. Hence, in total, the proposal debugging system induces an overhead of

$$
372+18 s+42 k
$$

bytes, where $k$ is the number of instructions of the original program.

For reference, a microcontroller such as the ATmega328P which we used in this experiment has 32 Kbytes (i.e. 32,768 bytes) of flash program memory (i.e. to store the machine code). So, the following inequality must be satisfied for the debugging system proposal to be applicable to the original program:

$$
372+18 s+42 k+2 k_{1}+4 k_{2} \leq 32768
$$

with $k$ the number of instructions of the original program, out of which $k_{1}$ 
instructions are single-word and $k_{2}$ double-word; so, $k_{1}+k_{2}=k$.

Besides, the required memory size depends on the number of original OUT instructions to the port D as explained in Section 4.2. Given that this port is almost reserved for the debugging system, we can assume that the occurrence of such an original instruction is low, so, for the sake of clarity, it is not included in the memory size calculation below. Furthermore, we can assume without loss of generality that the original program is made of single-word instructions (in fact, the only instructions that are double-word are the long jumps, long calls and very few, rather infrequent others; see for example Table 3). So, with, say, $s=111$ (111 instruction sorts, as listed in [30]), we obtain the following inequality with $k$ the number of instructions of the original program that can be tolerated given the size of the program memory:

$$
\begin{gathered}
372+18 s+42 k+2 k \leq 32768 \\
372+18 \times 111+44 k \leq 32768 \\
44 k \leq 30398 \\
k \leq 690
\end{gathered}
$$

Yet, the value of $s$ can be significantly reduced: a program hardly includes all the instruction sorts. A value of $s=40$ is arguably more meaningful (for instance, the initialisation code and the step code of the debugging system together merely rely on 21 instruction sorts, given in Appendix A, and moreover this code consists of non-trivial processes, such as interfacing the LCD); it induces $k \leq 719$. Given our assumptions, the order of the result rather than the exact value is meaningful.

Next, we measure the performance impact of the proposed debugging system. The values given below are counted in processor cycles, that is the number of clock ticks required to execute the corresponding machine code. Because the microcontroller used in this research, and thus in this experiment, is equipped with a processor clocked at $16 \mathrm{Mhz}$, such a number of cycles can be converted in milliseconds by dividing by $16 \times 10^{6}$. The initialisation code requires a total of $1,031,180$ cycles.

The number of cycles required by the step code depends on $t$ the time waited between each instruction (for the debugging information that is displayed on the LCD panel to be readable). In our implementation, $t$ is a multiple of approximately 1 second (1.00925 seconds, precisely), so for the sake of clarity, let us consider simply that $t$ is the delay in seconds. In our experiments, this delay is set to $t=1$.

Furthermore, the number of cycles of the step code depends on whether the corresponding original instruction is, for instance, the OUT instruction to the port D. In such case, 22 cycles are to be added; they correspond to the additional instructions injected as explained in Section 4.2. The same discussion is made for the other special cases (SBRC, etc.) as mentioned ibidem. But in total, the number of additional cycles induced by such special cases is extremely small compared to the total, and is thus negligible. So, for the sake of simplicity they 
are not included in the following discussion.

Assuming that for each instruction sort all the sixteen characters of the LCD panel are used to display the corresponding information, the step code requires a total of

$$
150601+16153080 t
$$

cycles. Again, this is an approximation: obviously, the magnitude of the number of cycles rather than the exact value is relevant.

So, in total, the proposed debugging system induces an overhead of

$$
1031180+k(150601+16153080 t)
$$

cycles, where $k$ is the number of instructions of the original program.

Inducing the most significant overhead, both memory and performance, we give in Appendix B the details of the overhead calculations. This also shows the step code that is injected in most cases by the proposed debugging system for each instruction of the original program.

\section{Discussion}

First and foremost, the various experimental results have demonstrated that we succeeded in realising a fully autonomous debugging system that is applicable to any sort of program created for an AVR microcontroller. The proposal thus effectively raises the human-machine interaction usability by providing real-time, in situ debugging information to the user, while retaining the cost of such a debugging process minimal, especially compared to the conventional approach that relies on a computer dedicated to debugging.

Furthermore, the results have shown that the proposal is applicable to sensor devices: two effective real-world sensors (a light sensor and a temperature sensor) were successfully realised and put to test with the proposed debugging system, triggering an electronic signal to lighten an on-board LED when the corresponding condition was satisfied.

The results of the analysis of the memory overhead induced by the proposed debugging system on the original microcontroller program have shown that the extra memory required stays acceptable as the debugging system can tolerate an original program with about 700 instructions, or more depending on the optimization of the debugger code.

In addition, it can be noted that the almost entirety (more than 99\%) of the cycles of the initialisation code and of the step code injected for debugging are induced by waiting times. These waiting times are necessary especially to communicate with the LCD panel as per the manufacturer requirements [31] and also to ensure a short pause between the successive debugging information outputs in order to avoid displaying them on the LCD panel in a too rapid manner.

Therefore, the performance overhead can be deemed less significant than the memory overhead, especially since this overhead sort is induced almost entirely by waiting times, and most notably (16 153080 cycles per instruction of the original program, which makes most of the waiting times) the delays for a short 
pause between the debugging information display outputs, and that those delays for the pauses are adjustable to a certain extent.

Regarding the memory overhead (less significantly the performance overhead), let us mention that it could be further reduced by using the 8-bit operation mode of the LCD panel used in this research. This would however be a trade-off with the number of microcontroller pins that are reserved for the debugger: the 8-bit operation mode of the LCD panel requires twice as many data bus pins (eight instead of four, precisely) [31].

In order to reduce the memory and performance overhead induced by the proposed debugging system, these eight data bus pins of the LCD need to be connected to one single port of the microcontroller so that the corresponding values (bits) can be set in one single OUT instruction. In the case of the ATmega328P microcontroller which was used in this study, this can only be the port $\mathrm{D}$ and the port $\mathrm{B}$ as the port $\mathrm{C}$ provides only seven pins. And, for instance, in the case of an Arduino Uno board, this can only be the port $\mathrm{D}$ since the other ports (B and C) only provide six pins (the port B has two pins that are reserved for other purposes and only six out the seven pins of the port $C$ are available). This could interfere with the bootloader though, so a fully erased, blank chip would be needed.

\section{Conclusions}

Minimal power consumption is a very common requirement for sensors and similar devices. This is the reason why they are often based on microcontrollers, with those based on the AVR processor architecture being very popular. In addition and by definition, sensors rely on specific hardware to gather various information. Specialised hardware means specialised software development and debugging. In this paper, we have proposed a novel approach to this issue by describing and evaluating a fully autonomous on-chip debugging solution for AVR microcontrollers, notably focusing on the human-machine interaction issue. In other words, we have shown with a constructive proof that an autonomous debugging mechanism can be implemented for sensors based on AVR microcontrollers with an optimally low-given the accessible hardware-number of dedicated (reserved) pins (six) and a very reasonable 42-byte per original instruction overhead of the program memory needed for the original, debugged program. The proposal has been validated in practice through various experiments and its overhead formally measured.

Regarding future works, although some refinements for the proposal might be planned, this ought to be carefully considered so that the overhead induced by the debugging system stays minimal, as it is in this research; this is about both the hardware (e.g. microcontroller pins) and software (e.g. memory) requirements. Nevertheless, possible improvements include for example simplification of the tool chain and documentation. In addition, it would be meaningful to analyse the portability of the proposed debugging solution to other microcontroller architectures. 


\section{Funding}

This research was partly supported by a Grant-in-Aid for Scientific Research (C) of the Japan Society for the Promotion of Science under grant no. 19K11887.

\section{Acknowledgements}

The author is grateful towards the reviewers for their comments and suggestions which helped to improve the paper. And he sincerely thanks Takeyuki Nagao (Chiba University of Commerce, Japan) for his advices on microcontrollers.

\section{Conflicts of Interest}

The author declares no conflicts of interest regarding the publication of this paper.

\section{References}

[1] Caroll, A. and Heiser, G. (2010) An Analysis of Power Consumption in a Smartphone. Proceedings of the 2010 USENIX Annual Technical Conference, Boston, 2225 June 2010, 271-284.

[2] Troglia Gamba, M., Nicola, M. and Motella, B. (2021) Computational Load Analysis of a Galileo OSNMA-Ready Receiver for ARM-Based Embedded Platforms. Sensors, 21, 467. https://doi.org/10.3390/s21020467

[3] Rohani, A. and Zarandi, H.R. (2009) An Analysis of Fault Effects and Propagations in AVR Microcontroller ATmega103(L). Proceedings of the International Conference on Availability, Reliability and Security, Fukuoka, 16-19 March 2009, 166-172. https://doi.org/10.1109/ARES.2009.169

[4] Claasen, T.A.C.M. (2006) An Industry Perspective on Current and Future State of the Art in System-on-Chip (SoC) Technology. Proceedings of the IEEE, 94, 1121 1137. https://doi.org/10.1109/JPROC.2006.873616

[5] Chen, Y.-K. and Kung, S.Y. (2008) Trend and Challenge on System-on-a-Chip Designs. Journal of Signal Processing Systems, 53, 217-229. https://doi.org/10.1007/s11265-007-0129-7

[6] Conti, G., Quintana, M., Malag, P. and Jimnez, D. (2020) An FPGA Based Tracking Implementation for Parkinson's Patients. Sensors (Basel), 20, 3189. https://doi.org/10.3390/s20113189

[7] Jiang, X.Y., Lu, Y.P., Tang, H.-Y., Tsai, J.M., Ng, E.J., Daneman, M.J., Boser, B.E. and Horsley, D.A. (2017) Monolithic Ultrasound Fingerprint Sensor. Microsystems \& Nanoengineering, 3, Article No. 17059.

https://doi.org/10.1038/micronano.2017.59

[8] Fortino, G., Giannantonio, R., Gravina, R., Kuryloski, P. and Jafari, R. (2013) Enabling Effective Programming and Flexible Management of Efficient Body Sensor Network Applications. IEEE Transactions on Human-Machine Systems, 43, 115-133. https://doi.org/10.1109/TSMCC.2012.2215852

[9] Richards, P. and Debugger, A. (2018). https://github.com/Paulware/ArduinoDebugger

[10] Simmons, S.J. (2020) UnoArduSim. Queen's University, Kingston. https://www.sites.google.com/site/unoardusim/home

[11] Arduino. Libraries. See the "Standard Libraries" Section. 
https://www.arduino.cc/reference/en/libraries

[12] Baker, Z.K. and Monson, J.S. (2009) In-Situ FPGA Debug Driven by On-Board Microcontroller. Proceedings of the 17 th IEEE Symposium on Field Programmable Custom Computing Machines, Napa, 5-7 April 2009, 219-222. https://doi.org/10.1109/FCCM.2009.9

[13] Bermbach, R. and Kupfer, M. (2006) Development of a Debug Module for a FPGABased Microcontroller. IFAC Proceedings Volumes, 39, 275-280. https://doi.org/10.1016/S1474-6670(17)30197-0

[14] Maier, K.D. (2003) On-Chip Debug Support for Embedded Systems-on-Chip. Proceedings of the International Symposium on Circuits and Systems, Bangkok, 25-28 May 2003, V-565-V-568.

[15] Sudeendra, K.K., Sahoo, S., Mahapatra, A., Swain, A.K. and Mahapatra, K.K. (2017) Security Enhancements to System on Chip Devices for IoT Perception Layer. Proceedings of the IEEE International Symposium on Nanoelectronic and Information Systems, Bhopal, 18-20 December 2017, 151-156.

[16] Hu, Y.L. and Xiong, B. (2006) Design of an Embedded On-Chip Debug Support Module of a MCU. Proceedings of the Conference on High Density Microsystem Design and Packaging and Component Failure Analysis, Shanghai, 27-28 June 2006, 5-8. https://doi.org/10.1109/HDP.2006.1707555

[17] Park, K., Yoo, S.G., Kim, T. and Kim, J. (2010) JTAG Security System Based on Credentials. Journal of Electronic Testing, 26, 549-557. https://doi.org/10.1007/s10836-010-5170-y

[18] Dolinay, J., Dostlek, P. and Vaek, V. (2016) Arduino Debugger. IEEE Embedded Systems Letters, 8, 85-88. https://doi.org/10.1109/LES.2016.2619692

[19] Rahman, H. and Arefin, M.T. (2019) Design and Analysis of an Experimental Data and Clock Multiplexing Technique for Generating Faster Single Wire Synchronous Data Bus. Proceedings of the 2 nd International Conference on Innovation in Engineering and Technology, Dhaka, 23-24 December 2019, 1-5. https://doi.org/10.1109/ICIET48527.2019.9290705

[20] Corke, P., Valencia, P., Sikka, P., Wark, T. and Overs, L. (2007) Long-Duration Solar-Powered Wireless Sensor Networks. Proceedings of the 4th Workshop on Embedded Networked Sensors, Cork, 25-26 June 2007, 33-37. https://doi.org/10.1145/1278972.1278980

[21] Wei, P., Ning, Z., Ye, S., Sun, L., Yang, F.H., Wong, K.C., Westerdahl, D. and Louie, P.K.K. (2018) Impact Analysis of Temperature and Humidity Conditions on Electrochemical Sensor Response in Ambient Air Quality Monitoring. Sensors, 18, 59. https://doi.org/10.3390/s18020059

[22] Microchip Technology (2020) Microchip Studio (4. Debugging). Revision D.

[23] Stallman, R., Pesch, R., Shebs, S., et al. (2017) Debugging with GDB-The GNU SourceLevel Debugger. Tenth Edition, Free Software Foundation, Boston.

[24] Microchip Technology (2017) Microchip Debugger (MDB) User's Guide. DS50002102D.

[25] Twin Earth Software (2017) Christophe Diericx. AVRDisassembler. https://github.com/twinearthsoftware/AVRDisassembler

[26] Sergeev, V.A. (2014) vAVRdisasm. https://github.com/vsergeev/vAVRdisasm

[27] Intel Corporation (1988) Hexadecimal Object File Format Specification. Revision A.

[28] IDA Pro. (2021) Hex-Rays. https://www.hex-rays.com/ida-pro 
[29] Microchip Technology (2020) AVR Instruction Set Manual. DS40002198A.

[30] Microchip Technology (2018) ATmega48A/PA/88A/PA/168A/PA/328/P megaAVR Data Sheet. DS40002061A.

[31] Hitachi (1998) HD44780U (LCD-II). ADE-207-272(Z), '99.9, Rev. 0.0. 


\section{Appendix A. The Instruction Sorts Used by the Proposed Debugging System (Initialisation Code and Step Code)}

As described in Section 6, the proposed debugging system can be optimised by adjusting the supported instruction sorts upon needs. This impacts the memory overhead induced by the debugging system. We state in Section 6 that a fraction of all the 111 instruction sorts is generally sufficient. This is further demonstrated in Table 1 and Table 2 which give the detail of the instruction sorts that are present inside the initialisation code and inside the step code of the proposed debugging system, respectively. In total, only 21 instruction sorts are present inside both programs. This in addition shows the non-pervasiveness of the proposal.

Table 1. The instruction sorts present in the initialisation code injected by the proposed debugging system.

\begin{tabular}{ccc}
\hline$\#$ & Instruction & Description \\
\hline 1 & andi & logical AND with immediate value \\
2 & breq & branch if equal \\
3 & brne & branch if not equal \\
4 & dec & decrement \\
5 & ldi & load immediate value \\
6 & lds & load from data memory space \\
7 & lpm & load from program memory space \\
8 & lsl & logical shift left \\
9 & lsr & logical shift right \\
10 & mov & copy register \\
11 & or & logical OR \\
12 & ori & logical OR with immediate value \\
13 & out & store to I/O location \\
14 & pop & pop from the stack \\
15 & push & push onto the stack \\
16 & rcall & relative subroutine call \\
17 & ret & return from subroutine \\
18 & rjmp & relative unconditional jump \\
19 & sts & store in data memory space \\
20 & & test for zero or minus \\
& & \\
\hline & tst &
\end{tabular}

Table 2. The instruction sorts present in the step code injected by the proposed debugging system.

\begin{tabular}{ccc}
\hline$\#$ & Instruction & Description \\
\hline 1 & brne & branch if equal \\
2 & dec & decrement \\
3 & in & read from I/O location \\
4 & ldi & load immediate value \\
5 & out & store to I/O location \\
6 & pop & pop from the stack \\
7 & rcall & relative subroutine call \\
8 & push & push onto the stack \\
\hline
\end{tabular}




\section{Appendix B. Details and Overhead Measurement for the Step Code Injected by the Proposed Debugging System}

As described in Section 4.2, the step code is automatically injected by the proposed debugging system for (almost) each instruction of the original program (i.e. the program that is being debugged). It is thus responsible for the majority of the performance overhead and of the memory overhead that are induced by the debugging system. Hence, it is meaningful to describe more in detail this part of the proposal, and to show how the overhead was calculated.

This is summarised in Table 3. The "Cycles" column indicates the number of cycles that are required by the instruction, whereas the "Total cycles" column indicates the total number of cycles required when executing the step code, that is taking into account that some instructions are executed several times because, for instance, of loops inside the program.

Table 3. Details and overhead measurement for the step code injected by the proposed debugging system.

\begin{tabular}{|c|c|c|c|c|c|}
\hline$\#$ & Instruction & Words & Bytes & Cycles & Total cycles \\
\hline 1 & push r0 & 1 & 2 & 2 & 2 \\
\hline 2 & in $\mathrm{r} 0$, SREG & 1 & 2 & 1 & 1 \\
\hline 3 & rcall .+CLEAR_CODE & 1 & 2 & 3 & 79,721 \\
\hline 4 & push r31 & 1 & 2 & 2 & 2 \\
\hline 5 & push r30 & 1 & 2 & 2 & 2 \\
\hline 6 & ; ldi instruction dynamically added & 1 & 2 & 1 & 1 \\
\hline 7 & ; ldi instruction dynamically added & 1 & 2 & 1 & 1 \\
\hline 8 & rcall .+PRINT_CODE & 1 & 2 & 3 & 70,856 \\
\hline 9 & pop r30 & 1 & 2 & 2 & 2 \\
\hline 10 & pop r31 & 1 & 2 & 2 & 2 \\
\hline 11 & push r16 & 1 & 2 & 2 & 2 \\
\hline 12 & push r25 & 1 & 2 & 2 & 2 \\
\hline 13 & ldi r16, 4; wait 4 times about $250 \mathrm{~ms}$ & 1 & 2 & 1 & 1 \\
\hline 14 & ldi r 25,250 & 1 & 2 & 1 & 4 \\
\hline 15 & rcall .+WAIT_CODE & 1 & 2 & 3 & $16,153,064$ \\
\hline 16 & dec r16 & 1 & 2 & 1 & 4 \\
\hline 17 & brne wait_loop & 1 & 2 & 1,2 & 7 \\
\hline 18 & pop r25 & 1 & 2 & 2 & 2 \\
\hline 19 & pop r16 & 1 & 2 & 2 & 2 \\
\hline 20 & out SREG, r0 & 1 & 2 & 1 & 1 \\
\hline \multirow[t]{2}{*}{21} & pop r0 & 1 & 2 & 2 & 2 \\
\hline & Total & 21 & 42 & - & $16,303,681$ \\
\hline
\end{tabular}

